\title{
Determination and Variation of Core Bacterial Community in a Two- Stage Full-Scale Anaerobic Reactor Treating High-Strength Pharmaceutical Wastewater ${ }^{\mathrm{S}}$
}

\author{
Haijun Ma, Lin Ye, Haidong Hu, Lulu Zhang, Lili Ding, and Hongqiang Ren* \\ State Key Laboratory of Pollution Control and Resource Reuse, School of the Environment, Nanjing University, Nanjing 210023, Jiangsu, \\ P.R. China
}

\section{Received: July 12, 2017 \\ Revised: August 15, 2017 \\ Accepted: August 23, 2017 \\ First published online \\ August 25, 2017 \\ ${ }^{*}$ Corresponding author \\ Phone: +86-25-89680512; \\ Fax: +86-25-89680569; \\ E-mail: hqren@nju.edu.cn}

S upplementary data for this paper are available on-line only at http://jmb.or.kr.

pISSN 1017-7825, eISSN 1738-8872

Copyright $(C) 2017$ by

The Korean Society for Microbiology and Biotechnology
Knowledge on the functional characteristics and temporal variation of anaerobic bacterial populations is important for better understanding of the microbial process of two-stage anaerobic reactors. However, owing to the high diversity of anaerobic bacteria, close attention should be prioritized to the frequently abundant bacteria that were defined as core bacteria and putatively functionally important. In this study, using MiSeq sequencing technology, the core bacterial community of 98 operational taxonomic units (OTUs) was determined in a twostage upflow blanket filter reactor treating pharmaceutical wastewater. The core bacterial community accounted for $61.66 \%$ of the total sequences and accurately predicted the sample location in the principal coordinates analysis scatter plot as the total bacterial OTUs did. The core bacterial community in the first-stage (FS) and second-stage (SS) reactors were generally distinct, in that the FS core bacterial community was indicated to be more related to a higherlevel fermentation process, and the SS core bacterial community contained more microbes in syntrophic cooperation with methanogens. Moreover, the different responses of the FS and SS core bacterial communities to the temperature shock and influent disturbance caused by solid contamination were fully investigated. Co-occurring analysis at the Order level implied that Bacteroidales, Selenomonadales, Anaerolineales, Syneristales, and Thermotogales might play key roles in anaerobic digestion due to their high abundance and tight correlation with other microbes. These findings advance our knowledge about the core bacterial community and its temporal variability for future comparative research and improvement of the two-stage anaerobic system operation.

Keywords: Anaerobic digestion, anaerobic bacteria, core bacterial community, network analysis

\section{Introduction}

Anaerobic digestion (AD) is the most prominent bioenergy technology and has been widely used for the treatment of various organic wastes such as municipal sludge, cellulose waste, and high-strength industrial wastewater [1]. AD is a highly complex biochemical process that depends on the well-balanced cooperation of four groups of microbes, including hydrolyzing bacteria, fermenting bacteria, specialized acetogenic syntrophs, and methanogenic archaea $[2,3]$. Owing to the successful separation of acidogenesis and methanogenesis, the two-stage anaerobic reactors can achieve better overall performance than the single-stage reactor and have a greater capacity to withstand environmental disturbance $[4,5]$. Thus, the two-stage anaerobic reactor has grown to be a promising process for organic waste treatment since its emergence [6].

To better understand the metabolic mechanisms involved in the $\mathrm{AD}$ process, characterization of the anaerobic microbes is of great importance. On account of the complex function related to hydrolysis, fermentation, and acetogenesis and at least two orders higher abundance in comparison 
with archaea, there is an urgent need to pay attention to the bacterial population in anaerobic reactors $[7,8]$. With the development of molecular techniques $[5,9,10]$, especially the appearance of next-generation sequencing technology [11], more and more efforts have been made to get insight into the bacterial community in anaerobic reactors to better understand the metabolic interaction involved in the AD process. Different bacterial profiles have been revealed in different anaerobic reactors [12-15]. Nevertheless, these indepth studies mainly focused on the single-stage anaerobic configuration, and not a great deal of information is available about the bacteria in the two-stage anaerobic reactors. Schievano et al. [5] detected a less diverse population of fermentative bacteria in the second-stage (SS) reactor than that in the first-stage (FS) reactor using PCR-DGGE. Kongjan et al. [6] demonstrated that the FS reactor was dominated with the hydrogen-producing bacteria Thermoanaerobacter wiegelii, Caldanaerobacter subteraneus, and Caloramator fervidus, whereas Methanosarcina mazei and Methanothermobacter were dominant in the SS reactor in a two-stage upflow anaerobic sludge blanket (UASB) reactor. However, these studies were based on lab-scale reactors. There is still a lack of knowledge about the microbial community in the full-scale two-stage anaerobic reactors.

Because of the high diversity of the anaerobic bacterial community, functional characterization of each bacterium is laborious. Close attention should be prioritized to the frequently abundant bacteria that were defined as core bacteria and putatively played key roles in the degradation of organic pollutants [16]. Riviere et al. [17] defined a core bacterial community of six OTUs accounting for $23 \%$ of the total sequences in anaerobic digesters. Similarly, Lee et al. [18] defined OTUs representing more than $3 \%$ of the sequences in each digester as the core bacterial community. Although these studies greatly improved our understanding about the core bacteria participating in the degradation of pollutants, the core bacterial community in two-stage anaerobic reactors remains unclear. Moreover, the bacterial community in anaerobic reactors could be influenced by environmental factors (e.g., $\mathrm{pH}$, temperature) [19] and then the change in microbial community structure would subsequently influence the performance of anaerobic reactors [2]. Thus, knowledge on the temporal variation and the response to environmental perturbations of the core bacterial community will be vital to the improvement and optimization of the microbial management of twostage anaerobic reactors.

This paper mainly focused on the determination and variability of the core bacterial community in two-stage anaerobic reactors by monitoring a full-scale mesophilic two-stage upflow blanket filter (UBF) reactors treating 100\% high-strength pharmaceutical wastewater over 9 months. Anaerobic sludge samples were temporally collected from both reactor stages and sequenced to characterize the microbial composition and variability in correlation to digester performance.

\section{Materials and Methods}

\section{Two-Stage UBF Reactor Performance and Sampling}

The monitored mesophilic two-stage UBF reactors have been operating in continuous flow since June 2013, consisting of 10 same UBF reactors (size: $\Phi 8 \mathrm{~m} \times 20 \mathrm{~m}$ ), among which four are operated in parallel as the FS reactors and the other six as the SS reactors. There is a regulating reservoir that has a volume of $1,200 \mathrm{~m}^{3}$ to connect the FS and SS reactors. These reactors were all originally inoculated with excess sludge from a full-scale wastewater treatment plant treating chemical industrial-park wastewater. The $\mathrm{pH}$ of the FS and SS reactors was maintained at $7.5 \pm 0.2$ and $7.8 \pm 0.2$, respectively, so that the UBF reactors were not operated for separating the acidogenesis and methanogenesis processes. After being pretreated by inclined plate sedimentation and dissolved air flotation, the influent was cleaned out of suspended solids. The system treats $2,400 \mathrm{~m}^{3}$ pharmaceutical wastewater every day, and the hydraulic retention time (HRT) of the FS and SS reactors is $40 \mathrm{~h}$ and $60 \mathrm{~h}$, respectively. All the reactors are operated at mesophilic conditons with the FS at $33 \pm$ $1^{\circ} \mathrm{C}$ and the SS at $35 \pm 1^{\circ} \mathrm{C}$. The chemical components in the influent are unclear and the biogas yield along with total suspended solids are unknown because the engineering configuration is a blocked system.

The chemical analysis for chemical oxygen demand (COD), $\mathrm{NH}_{4}{ }^{+}-\mathrm{N}$, temperature, and $\mathrm{pH}$ was conducted every day, and suspended solids were measured weekly. $\mathrm{COD}, \mathrm{NH}_{4}{ }^{+}-\mathrm{N}$, and suspended solids measurements were determined according to Standard Methods for the Examination of Water and Wastewater [20].

Twenty-one samples were temporally collected from $0 / 5 / 10 \mathrm{~m}$ in height of the 3rd FS reactor at 20/9/2014, 21/10/2014, 25/12/ 2014, 16/1/2015, 31/3/2015, 29/5/2015, and 23/7/2015. Additionally, 17 samples were taken from $0 \mathrm{~m}$ of the 5 th SS reactor at $20 / 9 / 2014,21 / 10 / 2014,25 / 12 / 2014,8 / 1 / 2015,11 / 1$ / 2015, 16/1/2015, 22/1/2015, 27/1/2015, 8/2/2015, 11/3/2015, 17/3/2015, 22/3/2015, 26/3/2015, 31/3/2015, 5/4/2015, 29/5/ 2015 , and $23 / 7 / 2015$. Before collecting the samples, we discharged the initial $20 \mathrm{~L}$ wastewater with anaerobic sludge to make sure that the residue in the sampling pipeline was washed out. Then, the samples were kept in an ice box and transported to the laboratory to centrifuge at $4,000 \times g$ for 5 min within the next $12 \mathrm{~h}$. The pellet of each sample was collected to be fixed with $50 \%$ alcohol (v/v) separately and stored at $-80^{\circ} \mathrm{C}$ until DNA extraction. 


\section{DNA Extraction, PCR, and Illumina MiSeq Sequencing}

After thawing at room temperature, total DNA of each sample was extracted from the pellet using the FastDNA Soil Kit (MP Biomedicals, USA) according to the standard protocol. The concentration and purity were determined by microspectrophotometry (NanoDrop ND-1000; NanoDrop Technologies, USA). Then, the extracted total DNA was stored at $-20^{\circ} \mathrm{C}$ before PCR amplification.

Twenty-four different pairs of bacterial primers $8 \mathrm{~F}\left(5^{\prime}\right.$-AGA GTTTGATYMTGGCTCAG-3') and 338R (5'-TGCTGCCTCCCG TAGGAGT-3') complemented with different 10-nt barcode sequences were designed to characterize the bacterial community. The barcode sequences were at least $2 \mathrm{nt}$ from each other so that every 24 samples with different primer sets could be pooled into one final sample as a single library for sequencing. Each PCR mixture included $5 \mu \mathrm{l}$ of $10 \times$ buffer, $40 \mathrm{ng}$ of DNA, $4 \mu \mathrm{l}$ of $2.5 \mathrm{mM}$ $\mathrm{dNTP}$ mixture, $4 \mu \mathrm{l}$ of $25 \mathrm{mM} \mathrm{MgCl}_{2}, 0.25 \mu \mathrm{l}$ of Ex Taq polymerase (Takara, Japan), $400 \mathrm{nM}$ of each primer, and $\mathrm{ddH}_{2} \mathrm{O}$ up to $50 \mu \mathrm{l}$. The PCR for bacterial amplification was carried out on a Veriti 96well thermal cycler (Applied Biosystems, USA) following the thermo-cycling procedure of initial denaturation at $95^{\circ} \mathrm{C}$ for $5 \mathrm{~min}$; followed by 20 cycles of $95^{\circ} \mathrm{C}$ for $30 \mathrm{sec}, 50^{\circ} \mathrm{C}$ for $50 \mathrm{sec}$, and $72^{\circ} \mathrm{C}$ for $40 \mathrm{sec}$; and a final $10 \mathrm{~min}$ extension at $72^{\circ} \mathrm{C}$. The $\mathrm{PCR}$ product was purified using the OMEGA E.Z.N.A Cycle-Pure Kit (Omega Bio-Tek, USA) according to the manufacturer's protocol. The concentration of the purified PCR product was determined with the Qubit 2.0 Fluorometer (Life Technologies, USA). Then, all 24 purified bacterial amplicons using different barcoded primers were pooled in equimolar amounts. Finally, we got two bacterial mixtures together with 10 other unknown bacterial amplicons for subsequent library construction. Finally, the two constructed libraries were sequenced on the MiSeq platform to generate 250bp paired-end reads.

\section{Bioinformatics, Statistical Analysis, and Data Availability}

The overall bioinformatics analysis was carried out using the Mothur software package (ver. 1.35.1) following the MiSeq SOP on the Mothur website (http://www.mothur.org/wiki/MiSeq_SOP) [21]. Detailed filtering steps are shown in the Methods of the Supplementary Information.

For analysis of bacterial OTUs, those representing no more than four sequences in total were excluded and we obtained 3,937 OTUs for downstream analysis. The similarities of bacterial community patterns were determined by cluster analysis, which was conducted based on Bray-Curtis distances using PAST3 [22]. All the 38 samples were divided into four groups based on the cluster analysis result. The visualization of microbial communities in different groups was conducted using Circos [23]. Principal coordinates analysis (PCoA) was conducted based on all the bacterial OTUs and the 98 core bacterial OTUs, respectively. STAMP was applied to identify the families that were differently abundant in different groups [24]. The orders co-occurrence patterns were tested with the checkerboard score (C-score) under no interactions between different phylogenetic taxa, in which a Cscore unit is a $2 \times 2$ matrix where both orders (orders in the core bacterial community) occur once but on different sites [25]. A cooccurrence event was confirmed to be valid if the Spearman's coefficient $(\rho)$ was both $>0.6$ and statistically significant ( $p$-value $<0.01$ ) [26]. Network visualization was conducted using the interactive platform Gephi (WebAtlas, France) with Fruchterman Reingold placement algorithm [27].

Sequence data used in this study have been submitted to the Sequence Read Archive with the accession number PRJNA331231.

\section{Results and Discussion}

\section{Reactor Performance}

As pharmaceutical factories are mainly engaged in producing antibiotic pills by culturing functional bacteria, the original wastewater contains mainly culture medium and rare cellulose. Owing to the changing production plan according to the market, the COD of the wastewater influent varied in a wide range from 4,080 to $14,027 \mathrm{mg} / 1$ (Fig. 1A). To ensure stable performance of the digesters, the COD concentration of influent wastewater was maintained below 10,000 mg/l after late December 2014. The COD removal of both FS and SS reactors remained relatively steady at around $50 \%$ and $60 \%$, respectively (Fig. 1), so the overall COD removal was approximately $80 \%$. Chen et al.

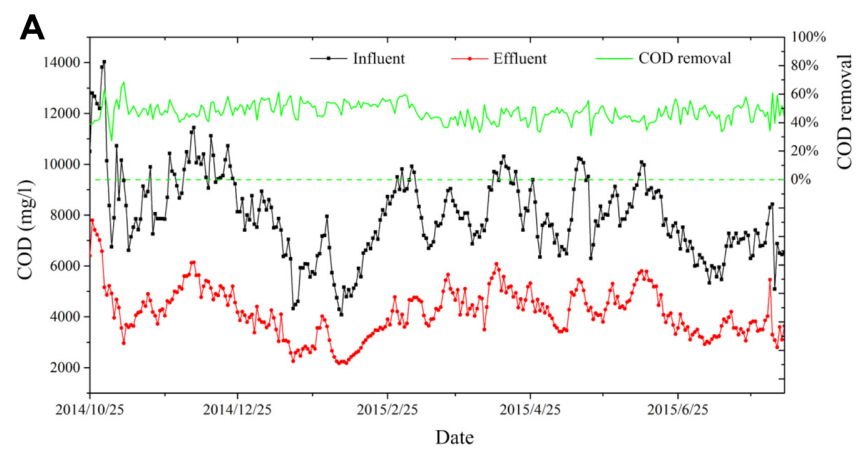

B

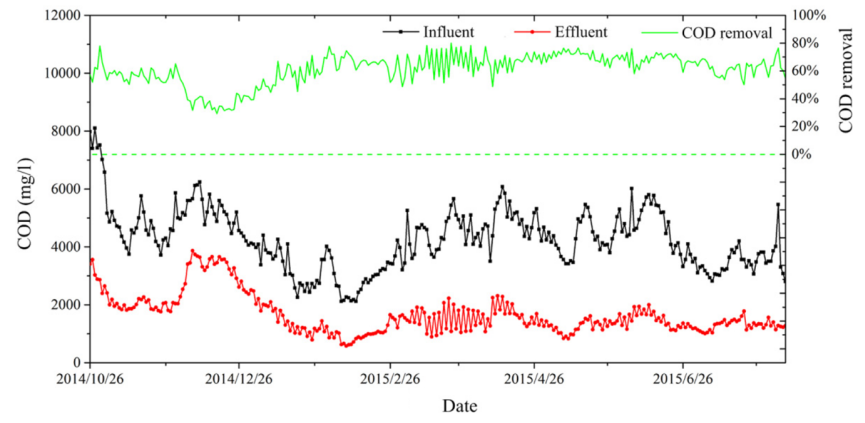

Fig. 1. COD of the influent and effluent, and COD removal, in the (A) first-stage and (B) second-stage reactors. 
[28] reported that the average removal was $63.3 \%$ in a fullscale UASB reactor treating chemical synthesis-based pharmaceutical wastewater containing 6-aminopenicillanic acid and amoxicillin. The relatively lower COD removal might be attributed to chemicals in the synthesis-based wastewater having greater toxic effects on the anaerobic microbes. In addition, the alkalinity was about $3 \mathrm{~g} \mathrm{CaCO}_{3} / 1$ and the $\mathrm{pH}$ in the reactors varied between 7.3 and 8.0. This indicated that the whole reactors showed generally stable performance.

There had been two perturbations during the study period (20/9/2014-8/8/2015). The first perturbation occurred at the middle of December 2014, on account of the influent being over heated from $31^{\circ} \mathrm{C}$ to about $36^{\circ} \mathrm{C}$. Therefore, the temperature of the wastewater was gradually increased to $38 \pm 1^{\circ} \mathrm{C}$ in the FS reactors and to $40 \pm 1^{\circ} \mathrm{C}$ in the SS reactors. The COD removal of the FS reactors remained generally stable during the disturbance. However, the COD removal of the SS reactors decreased significantly to $29.2 \%$ (Fig. 1B). After changing the temperature of the influent to around $31^{\circ} \mathrm{C}$ in about 5 days, the performance of the SS reactors gradually recovered from the temperature shock. The second perturbation happened at late March 2015 due to the breakdown of air flotation pretreatment. As a result, many undissolved contaminants flowed into the FS reactors along with the influent. The non-bioavailable part of the undissolved pollutants (e.g., sands) with high settling velocity started to accumulate quickly at the bottom of the FS reactors and served as inert carriers for biomass, partly similar to an anaerobic filter reactor [29]. Thus, anaerobic sludge began to grow and the suspended solids at $0 \mathrm{~m}$ of the FS reactors increased rapidly to $120 \mathrm{~g} / 1$ within 2 weeks. However, the performance of the FS and SS reactors seemed not to be disturbed (Fig. 1).

\section{Determination of Core Bacterial Community}

The bacterial community composition of the entire anaerobic sludge was identified using barcoded amplicons generating at least 20,546 clean sequences per sample, so all the bacterial datasets were normalized to 20,546 sequences for subsequent analysis. The sequences of all bacterial amplicons were clustered into OTUs at 6\% cutoff together. The proportion of the sequences in each OTU was regarded as the practical numerical abundance of that organism for simplicity, even though the observed read abundance were probably distorted by different DNA extraction efficiencies and differing $16 \mathrm{~S}$ rRNA gene copy numbers of each organism and various barcoded primers [16]. After discarding OTUs representing fewer than four sequences in total, the number of OTUs decreased from 19,037 to 3,937 to greatly speed up downstream OTU analysis. The proportion of remaining sequences within each amplicon varied between $92.82 \%$ and $98.83 \%$ (data not shown).

To determine the core bacterial community in the anaerobic reactors, we counted the frequency and read abundance of all the bacterial OTUs. A few frequently observed OTUs took up most of the reads (Fig. S1). The 73 OTUs common to all 38 samples occupied $53.92 \%$ of the total sequences, and OTUs observed in more than 30 samples accounted for $77.97 \%$ of the total sequences. However, the 2,325 OTUs observed in 10 and fewer samples only made up $3.75 \%$ of the total sequences.

Due to the fact that shared OTUs across all samples would be affected by the sequencing depth, simply analyzing the frequently observed OTUs would be less informative for determination of the core community. For example, among the 72 OTUs common to all samples, the top 10 least OTUs only accounted for $0.61 \%$ of the total sequences when ranked by decreasing abundance. These low abundance but commonly shared OTUs only represented a few sequences in most samples and were sensitive to sequencing depth. In addition, a few abundant OTUs represented the majority of all reads in each sample. To avoid deviation of the core community, we focused on the abundant OTUs that were defined as the top OTUs making up $80 \%$ of the reads in a sample when ranked by decreasing abundance [16]. The proportion of the sequences belonging to all the 532 abundant bacterial OTUs out of 3,937 OTUs reached up to $88.98 \%$ of total sequences. On account of the bacterial community structure in the FS and SS reactors being functionally different, the abundant bacterial OTUs were divided into four groups based on the frequency with which they were abundant in FS and SS samples. Group 1 contained 12 OTUs, which were abundant in more than 17 FS samples ( $80 \%$ of all FS samples) and more than 14 SS samples ( $80 \%$ of all SS samples) at the same time. These 12 OTUs made up $26.12 \%$ of the total reads. Group 2 consisted of 13 OTUs, which were abundant in more than 18 FS samples but fewer than 14 SS samples and accounted for $8.69 \%$ of the total reads. Group 3 OTUs were abundant in more than 14 SS samples but fewer than 18 FS samples and made up $26.85 \%$ of the total reads. The rest of the 434 abundant bacterial OTUs in group 4 were generally in much lower abundance than the other three groups and made up $27.33 \%$ of the total reads.

All 98 abundant bacterial OTUs in groups 1, 2, and 3 only made up $2.55 \%$ of all the original OTUs (3,937 OTUs), but accounted for $61.66 \%$ of the total reads, implying that these 
few kinds of organisms in the microbial community were of significant importance to pollutant degradation. These 98 OTUs constituted the core bacterial community in this two-stage UBF reactor. The core bacterial community contained 27 identified orders and 18 unclassified OTUs at the Order level.

\section{Generally Distinct Core Bacterial Community between FS and SS Reactors}

In order to determine the temporal variation of the bacterial community, PCoA was conducted based on all the bacterial OTUs. PCo1 and PCo2 made up $63.3 \%$ of the variation in the bacterial community structures (Fig. 2A). All 38 samples were grouped into four clusters based on cluster analysis at the division criteria that the similarity between any two samples from two different clusters was lower than 0.3 and similarity between any two sample from the same cluster was larger than 0.3 (Fig. S2). Then, the four clusters were indicated by four dotted ellipses in the PCoA scatter plot (Fig. 2). All the SS amplicons, excluding the three SS samples collected at 5/4/2015, 29/5/2015, and $23 / 7 / 2015$, fell into the same tight cluster (referred to as cluster I) located at the left of the scatter plot, indicating a highly consistent community structure in the SS reactors over the half-year period. Most FS samples were found in two other distinguished clusters, among which cluster II included FS samples obtained before 21/10/2014 and cluster III contained all the other FS samples except two samples collected from the bottom of FS reactors at 29/5/ 2015 and 23/7/2015. The last cluster (referred to as cluster IV) consisted of the remaining two FS samples and three SS samples. Moreover, the PCoA scatter plot according to all 98 core bacterial OTUs gave the same four clusters on the bacterial community composition of all the samples (Fig. 2B). Moreover, $70.4 \%$ of the variation in the core bacterial community of all the samples was explained by PCo1 and PCo2, which was quite close to $63.3 \%$ of the variation in the whole bacterial community. This similarity
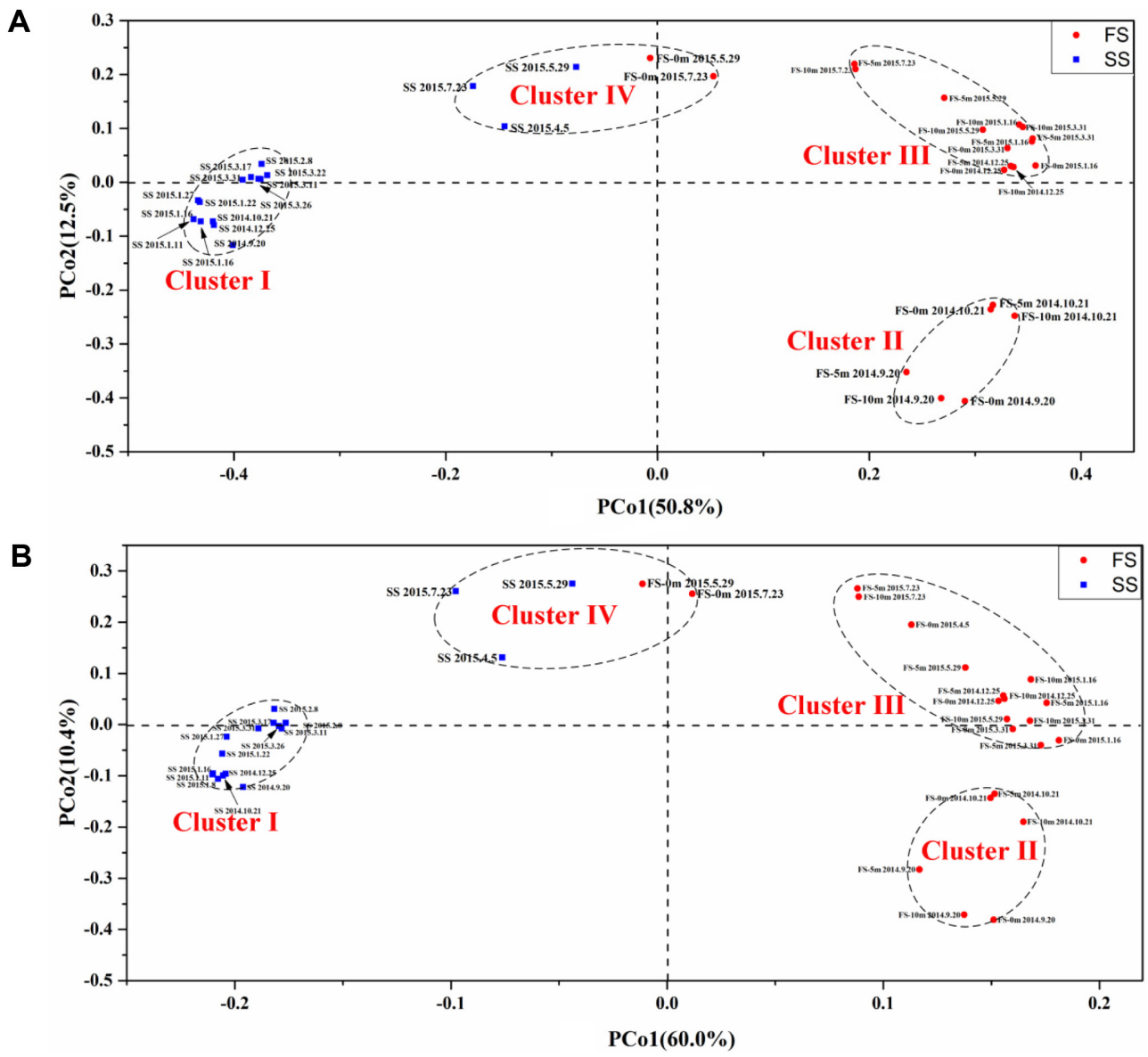

Fig. 2. Scatter plot of the first two PCos from principal coordinates analysis of the bacterial community in all samples based on (A) all the bacterial OTUs and (B) 98 core bacterial OTUs. 
in the patterns of the scatter plots for all bacterial OTUs and all core bacterial OTUs indicated that the core bacterial OTUs could accurately predict the sample location on the scatter plot and represented all the bacterial OTUs well.

According to the cluster result of PCoA on the core bacterial OTUs, all 38 samples were divided into four groups, referred to as SS-original, FS-original, FS-middle, and FS\&SS-final corresponding to clusters I, II, III, and IV. Bacteroidetes, Firmicutes, Proteobacteria, Chloroflexi, Synergistetes, and Spirochaetae were found to be prevalent in the core bacterial community in all the groups (Fig. 3), but the proportion of these phyla varied across the four groups (Table S1). Riviere et al. [17] investigated seven fullscale anaerobic digesters in France, Germany, and Chile

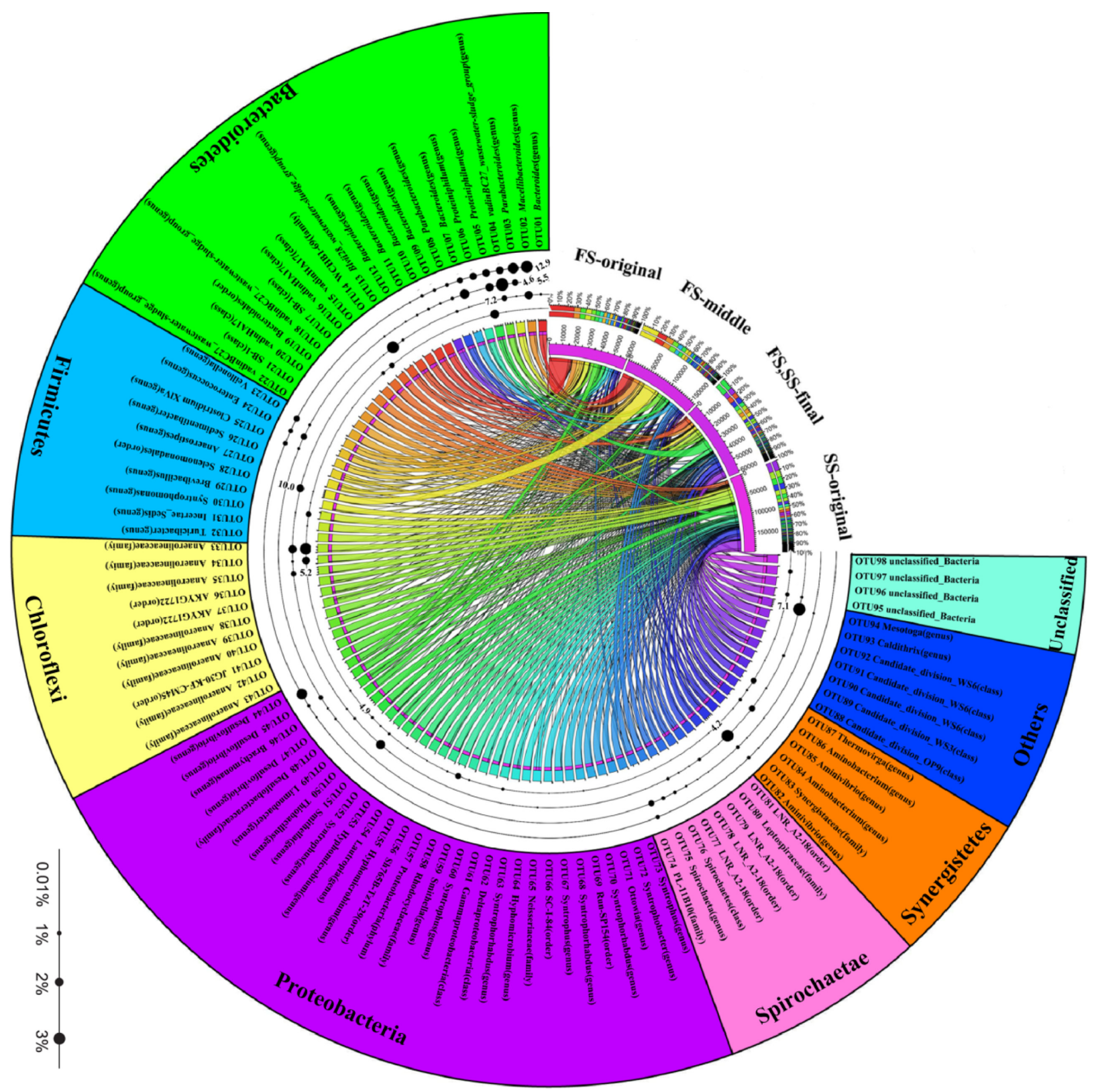

Fig. 3. Circular representation of the 98 core abundant bacterial OTUs in the four groups divided based on principle coordinates analysis.

The inner bubble plot shows the average relative abundance of every OTU in each group. The bubble plot comprises four layers, which from outside to inside represent first-stage (FS)-original, FS-middle, FS \& second-stage (SS)-final, and SS-original. The OTU labels are the lowest assigned taxonomic rank. The percentages $\leq 4 \%$ are indicated by dots with different sizes and the percentages $\geq 4 \%$ are shown as numbers. 
and found bacteria within Proteobacteria, Bacteroidetes, Firmicutes, Chloroflexi, Synergistetes, and Spirochaetae to be the prevalent phenotypes in $\mathrm{AD}$, which was in agreement with the results of the present study.

Setting aside the two FS samples and three SS samples in group FS\&SS-final, the core bacterial community in the FS and SS reactors was generally distinct from each other (Fig. 2B). The most apparent difference between the core bacterial community in the FS and SS reactors was the different abundance of Bacteroidetes, Proteobacteria, and Chloroflexi (Table S1). The proportion of Bacteroidetes within the core bacterial community in the FS reactor (FSoriginal: average \pm deviation $=32.89 \pm 1.85 \%$; FS-middle: $27.25 \pm 5.89 \%$ ) was significantly higher than that in the SS reactor (SS-original: $10.02 \pm 2.61 \%$ ) (Table S1). The major members responsible for this difference in Bacteroidetes were Bacteroides, Macellibacteroides, Parabacteroides, and vadinBC27_wastewater-sludge_group (Fig. 3). Bacteroides was affiliated to family Bacteroidaceae, which possessed the capacity to degrade cellulose [30]. Both Macellibacteroides and Parabacteroides are members of family Porphyromonadaceae. Maspolim et al. [4] reported that Porphyromonadaceae was 1.6-2.4 times higher in relative abundance in the acidogenic reactor in comparison with the methanogenic reactor, implying that Porphyromonadaceae was related to higher fermentation steps in the $\mathrm{AD}$ process. The Proteobacteria in the SS reactor appeared to be more abundant than that in the FS reactor (Table S1). The Proteobacteria in the core bacterial community of the FS reactor primarily consisted of genus Desulfovibrio, whereas the proportion of many other syntrophic consortia, such as Smithella, Syntrophorhabdus, and Syntrophus, was higher in the SS reactor, implying more syntrophic metabolic pathways in the SS reactor. Chloroflexi was rare in the FS reactor but abundant in the SS reactor (Table S1). Of the sequences within Chloroflexi in the core bacterial community, 96.04\% were classified to family Anaerolineaceae, which can ultilize short-chain fatty acids as electron donors [31]. Concerning the Synergistetes, this phylum contains only one family, Synergistaceae. Members of this family were more abundant in the SS reactor (Table S1). Microbes affiliated to Synergistetes were proved to have the capability to ferment amino acids to volatile fatty acid [32]. Generally speaking, the core bacterial community in the FS reactor was correlated to a higher fermentation process. Nevertheless, the core bacterial community in the SS reactor contained many more microorganisms capable of syntrophic metabolic function, in cooperation with the methanogenic archaea [33].

\section{Core Bacterial Community in the FS and SS Reactors Responded Differently to the Two Perturbations}

The first perturbation of temperature shock occurred at the middle of December 2014, and the second perturbation at late March 2015 assigned all the FS samples into the three different groups FS-orginal, FS-middle, and FS\&SSfinal. However, the SS samples were only divided into two groups named SS-original and FS\&SS-final by the second perturbation. The core bacterial community in the FS reactor shifted in response to the increase of temperature, whereas the core bacterial community in the SS reactor remained stable through the first perturbation of temperature shock. On the contrary, the performance of the FS reactor remained undisturbed, but the COD removal of the SS reactor was drastically decreased to $29.2 \%$ (Fig. 1B). It seemed that the microorganisms in the FS reactor could survive the temperature shock without affecting the COD removal efficiency through changing the microbial community composition. The most variable family between the FS-original and FS-middle groups was the unclassified Selenomonadales (Fig. 4A). The proportion of the unclassified Selenomonadales increased from $0.11 \%$ to $10.22 \%$ after the temperature shock. It was reported that Selenomonadales was dominant at a lab-scale UASB reactor treating trichloroethylene wastewater [34]. Nevertheless, the metabolic function of Selenomonadales in the anaerobic system needs further investigation. Temperature was reported to be the major variable influencing the anaerobic microbial community structure [7, 18]. Werner et al. [14] found that the process temperature was a statistically important factor affecting the temporal shift of anaerobic bacterial community, which was in agreement with the present study. However, the core bacterial community in the SS reactor remained stable instead of shifting. The remarkable decrease and subsequent recovery of COD removal in the SS reactor was probably due to the significant inhibition, rather than functional failure, of microbial activity of the bacterial community in the SS reactor. The different response of the core bacterial community in the FS and SS reactors to the temperature shock was highly likely to be derived from their different community characteristics. The core bacterial community in the FS reactor was dominated by Bacteroidetes (Table S1), widely known as higher-level fermenter populations in the AD process. The microorganisms affiliated with this phylum primarily depend on undergoing competition of different origanisms that are capable of similar function to maintain functional stability [14]. On the other hand, there seemed to be many resistant populations in the SS reactor that could withstand disturbance without 


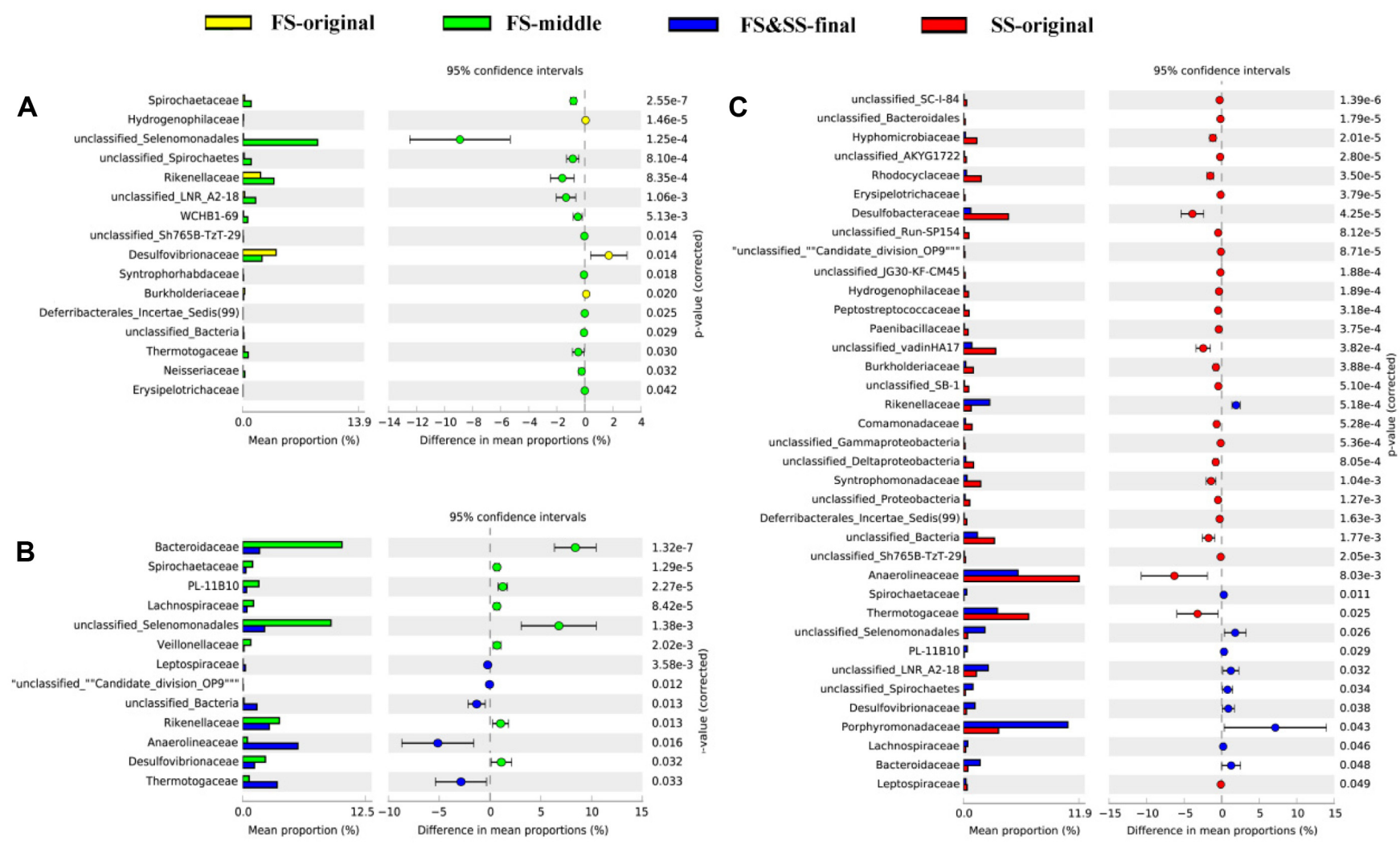

Fig. 4. Average abundance of families in the core bacterial community that were significantly differently abundant between pairs of groups.

(A) Groups first-stage (FS)-original and FS-middle; (B) groups FS-middle and FS \& second-stage (SS)-final; (C) groups FS\&SS-final and SSoriginal.

changes in community composition. As a result, the bacterial community in the SS reactors turned out to be undisturbed through the short temperature shock. Nowadays, the resistant populations in AD systems are still poorly understood and more work needs to be done to get deeper insight into the resistant microbiome by molecular biology methods [35].

The performance of both the FS and SS reactors survived through the second perturbation caused by the failure of air flotation pretreatment at late March 2015, but the bacterial community in both reactors shifted in response (Fig. 2B). After this disturbance, anaerobic sludge started to accumulate quickly at the bottom of the FS reactor, along with more and more undissolved suspended solids settling down. Interestingly, the five samples collected from the bottom of both the FS and SS reactors after the second perturbation were clustered together, demonstrating that the core bacterial community at the bottom of the FS and SS reactors shared the same variation trend to become similar to each other (Fig. 2B). However, the bacterial community at 5 and $10 \mathrm{~m}$ in the FS reactor remained stable as before.
The most remarkable change in the composition of the core community at the bottom of the FS reactor was the dramatic reduction in the proportion of Bacteroidaceae and unclassified Selenomonadales (Fig. 4B). Members in Bacteroidaceae are able to decompose carbohydrates, peptone, or metabolic intermediates, producing detectable organic acids [36]. There was a significant increase in the percentage of Anaerolineaceae and Thermotogaceae in the core bacterial community at the bottom of the FS reactor, whereas the proportion of these two families in the SS core bacterial community was nearly halved after the second perturbation (Figs. 4B and 4C). Members affliated to Thermotogaceae are strict anaerobes that are capable of fermenting many kinds of substrates into hydrogen and organic acids [37]. Anaerolineaceae was demonstrated to be the main consumers of short-chain fatty acids, indicating syntrophic cooperation with methanogenic archaea [31, 38]. The change of core bacterial community at the bottom of the FS reactor suggested more Anaerolineaceae and Thermotogaceae members along with the accumulation of 
anaerobic sludge, implying that a longer sludge retention time promoted the growth of Anaerolineaceae and Thermotogaceae. The core bacterial community at 5 and $10 \mathrm{~m}$ remained stable probably due to the fact that the anaerobic sludge only accumulated at the bottom of the FS reactor and made no difference to the bacterial community at 5 and $10 \mathrm{~m}$. Another notable change in the SS core bacterial community was that the proportion of family Porphyromonadaceae increased from $3.99 \%$ to $10.92 \%$ after the second perturbation (Fig. 4C). Porphyromonadaceae was reported to possess the capacity to degrade xylose, glucose, peptone, rhamnose, raffinose, saccharose, mannose, lactose, maltose, and cellobiose [36]. In general, the most variable families in the core bacterial community in the FS and SS reactors were Bacteroidaceae, unclassified Selenomonadales, Anaerolineaceae, Thermotogaceae, and Porphyromonadaceae.

It seemed that the two-stage $\mathrm{AD}$ system was running as a single-stage UBF reactor. Maspolim et al. [4] reported a few unique hydrolytic and acidogenic bacterial populations, including Porphyromonadaceae, Prevotellaceae, Ruminococcaceae, and unclassified Bacteroidetes in the FS reactor in comparison with the SS reactor. Nevertheless, Lee et al. [18] demonstrated that there was no apparent difference in bacterial community composition between the one-stage and two-stage digesters. The opposite findings were possibly due to their different operation conditions, as Maspolim et al. kept the FS and SS reactor at acidogenic and methanogenic conditions, respectively, whereas the investigated two-stage anaerobic reactors were not run for separating acid production and methane production in the study reported by Lee et al. Perhaps because the anaerobic system was not operated for separating the acidogenesis and methanogenesis processes in the present study, the operating parameter of the FS and SS reactors were generally the same except for the influent components and HRT. Once biomass succeeded to retain in the FS reactor by granulation, the generally consistent operating conditions tended to shape the same bacterial community structure in the FS and SS reactors regardless of the different influent and HRT.

\section{Network Analysis}

Correlation networks of co-occurring microbes enables the visualization of much valuable information [39] and have been successfully employed to explore the relationship patterns in soil microbial communities and marine microorganisms [26]. To discern the key bacterial taxa, the co-occurrence analysis was conducted based on the
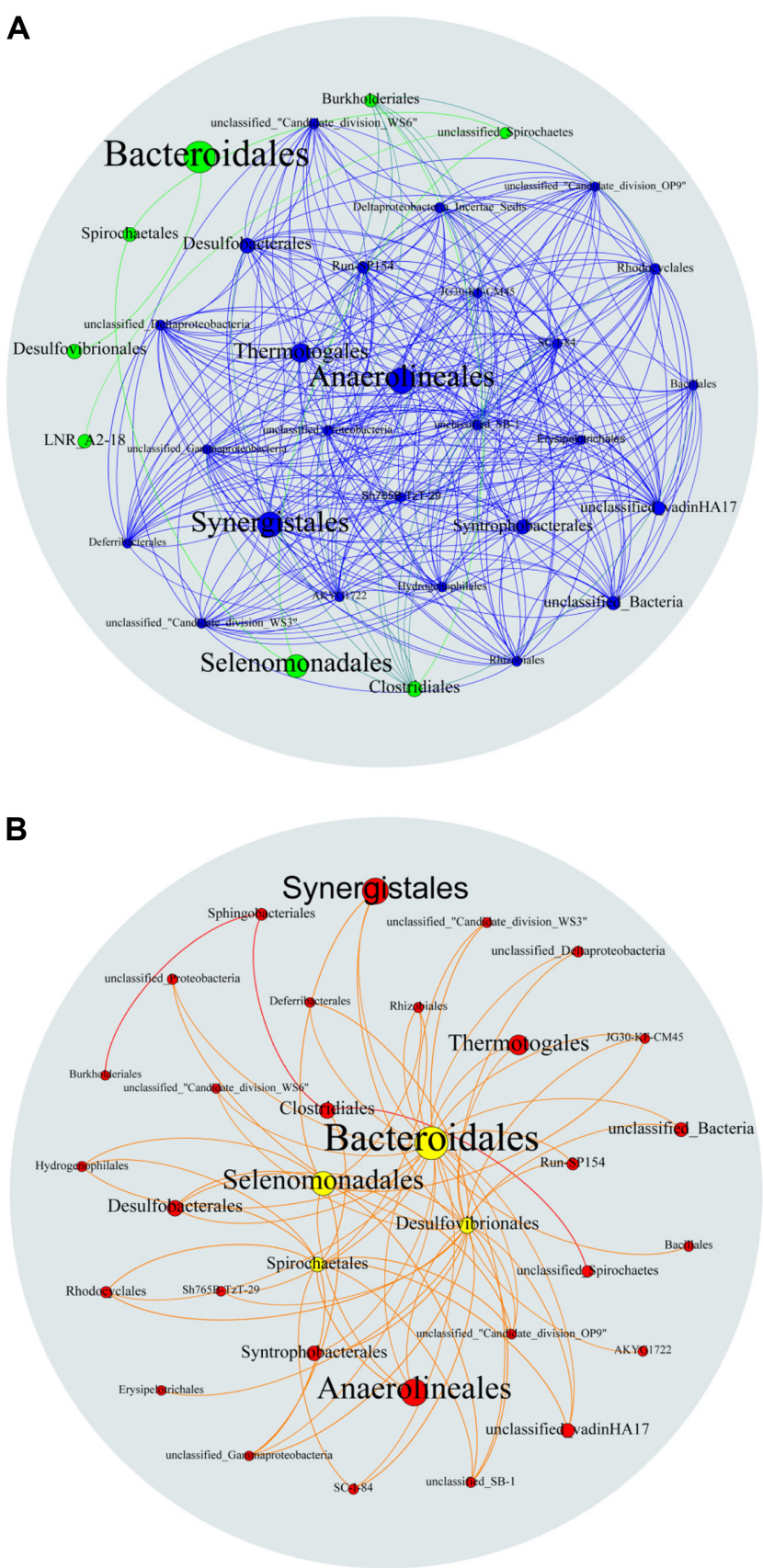

Fig. 5. Network analysis of co-occurrence of bacterial orders based on the classification profile of the core abundant bacterial OTUs.

A connection stands for a strong (Spearmans's $\rho>0.6$ or $\rho<-0.6$ ) and significant ( $p$-value $<0.05$ ) correlation. The size of each node is linear to the relative abundance of each order. (A) Network of positive correlation; the poorly connected nodes (fewer than 8 connections) are colored in green and the other highly connected nodes are colored in blue. (B) Network of negative correlation; the poorly connected nodes (fewer than 8 connections) are colored in red and the other highly connected nodes are colored in yellow. 
community pattern of the 98 core bacterial OTUs at the Order level. As a result, 319 robust positive correlations and 68 robust negative correlations were observed between 33 bacterial orders. The other four orders of low abundance showed no significant correlation with other orders. Bacteroidales was the most abundant order with only one positive correlation with Desulfovibrionales, and Selenomonadales had only two positive correlations with Spirochaetales and unclassified_Spirochaetes (Fig. 5A). There was a clear separation between the poorly connected nodes in green and the highly connected nodes in blue. Bacteroidales and Selenomonadales could probably accomplish the decomposition of organic matter by themselves, demanding little need for cooperation with other microbes. As for the two most abundant and variable orders in the FS core bacterial community (Table S2), these two orders were deduced to play an important role in the fermentation step in the $\mathrm{AD}$ process, indicating that the fermentation process was highly functionally independent. On the other hand, Bacteroidales had significantly strong negative correlation (23 negative connections) with other orders (Fig. 5B), implying potential competitive relationships with other bacteria. Unlike Bacteroidales, Selenomonadales, which is affliated to phylum Firmicutes, showed moderate negative correlation (9 negative connections) with other orders (Fig. 5B), suggesting that members assigned to this order were not easy to be affected by other microorganisms. Most orders were highly positively and poorly negatively connected to each other. These orders were found to be more abundant in the SS reactors (Table S2) and their highdegree association with each other indicated their tight functional cooperation. Among them, Anaerolineales, Syneristales, and Thermotogales were the three most abundant orders. Syneristales and Thermotogales were previously proved to be able to produce hydrogen and short-chain fatty acids, and Anaerolineales was reported to be the main consumers of volatile fatty acid [38]. It seemed that the production and consumption of short-chain fatty acids are required for the syntrophic cooperation of various microbes. On account of the abundance of microorganisms being closely related to their function attributes in anaerobic digesters [40], Bacteroidales, Selenomonadales, Anaerolineales, Syneristales, and Thermotogales could be the potential core orders to play a key role in the AD process.

In conclusion, sequences within Bacteroidetes, Firmicutes, Proteobacteria, Chloroflexi, Synergistetes, and Spirochaetae were found to be most abundant in the present two-stage UBF system. Out of 3,937 OTUs, 98 OTUs accounted for $61.84 \%$ of the total reads, constituting the core bacterial community and well representing the whole bacterial community. The FS core bacterial community was associated with higher fermentation steps in the $\mathrm{AD}$ process and the SS core bacterial community contained many more microbes capable of syntrophic cooperation with methanogens. The core bacterial community in the FS reactor was easy to be disturbed by high temperature shock, whereas SS core community remained stable. Both of the bacterial community structures in the FS and SS reactors shifted to be similar to each other after the second perturbation, probably due to the continuous operation for no separation of acidogenic and methanogenic processes. The major variable families in the FS and SS reactors through both perturbations were Bacteroidaceae, unclassified Selenomonadales, Anaerolineaceae, Thermotogaceae, and Porphyromonadaceae. Most importantly, co-occurrence analysis of the core bacterial community at the Order level suggested that Bacteroidales, Selenomonadales, Anaerolineales, Syneristales, and Thermotogales could probably play the key roles in AD and had the potential to be the monitoring microorganisms. With more detailed operation parameters combined with continuous monitoring of the microbial population, the adaption and response of the microbial community to environmental fluctuation could be more comprehensively understood.

\section{Acknowledgments}

This work was financially supported by the National Science \& Technology Support Program of China (No. 2014BAC08B04), and the Project of Jiangsu Provincial Six Talent Peaks (2015-JNHB-002).

\section{References}

1. van Lier JB, van der Zee FP, Frijters CTMJ, Ersahin ME. 2015. Celebrating 40 years anaerobic sludge bed reactors for industrial wastewater treatment. Rev. Environ. Sci. Biotechnol. 14: 681-702.

2. Briones A, Raskin L. 2003. Diversity and dynamics of microbial communities in engineered environments and their implications for process stability. Curr. Opin. Biotechnol. 14: $270-276$.

3. Chen Y, Cheng JJ, Creamer KS. 2008. Inhibition of anaerobic digestion process: a review. Bioresour. Technol. 99: 4044-4064.

4. Maspolim Y, Zhou Y, Guo C, Xiao K, Ng WJ. 2015. Determination of the archaeal and bacterial communities in two-phase and single-stage anaerobic systems by 454 pyrosequencing. J. Environ. Sci. 36: 121-129.

5. Schievano A, Tenca A, Scaglia B, Merlino G, Rizzi A, et al. 2012. Two-stage vs single-stage thermophilic anaerobic 
digestion: comparison of energy production and biodegradation efficiencies. Environ. Sci. Technol. 46: 8502-8510.

6. Kongjan P, O-Thong S, Angelidaki I. 2011. Performance and microbial community analysis of two-stage process with extreme thermophilic hydrogen and thermophilic methane production from hydrolysate in UASB reactors. Bioresour. Technol. 102: 4028-4035.

7. Sundberg C, Al-Soud WA, Larsson M, Alm E, Yekta SS, Svensson BH, et al. 2013. 454 Pyrosequencing analyses of bacterial and archaeal richness in 21 full-scale biogas digesters. FEMS Microbiol. Ecol. 85: 612-626.

8. Gonzalez-Estrella J, Sierra-Alvarez R, Field JA. 2013. Toxicity assessment of inorganic nanoparticles to acetoclastic and hydrogenotrophic methanogenic activity in anaerobic granular sludge. J. Hazard. Mater. 260: 278-285.

9. Talbot G, Topp E, Palin MF, Masse DI. 2008. Evaluation of molecular methods used for establishing the interactions and functions of microorganisms in anaerobic bioreactors. Water Res. 42: 513-537.

10. Rademacher A, Nolte C, Schoenberg M, Klocke M. 2012. Temperature increases from 55 to $75^{\circ} \mathrm{C}$ in a two-phase biogas reactor result in fundamental alterations within the bacterial and archaeal community structure. Appl. Microbiol. Biotechnol. 96: 565-576.

11. Vanwonterghem I, Jensen PD, Ho DP, Batstone DJ, Tyson GW. 2014. Linking microbial community structure, interactions and function in anaerobic digesters using new molecular techniques. Curr. Opin. Biotechnol. 27: 55-64.

12. Abendroth C, Vilanova C, Guenther T, Luschnig O, Porcar M. 2015. Eubacteria and archaea communities in seven mesophile anaerobic digester plants in Germany. Biotechnol. Biofuels 8: 87.

13. Nelson MC, Morrison M, Yu Z. 2011. A meta-analysis of the microbial diversity observed in anaerobic digesters. Bioresour. Technol. 102: 3730-3739.

14. Werner JJ, Knights D, Garcia ML, Scalfone NB, Smith S, Yarasheski K, et al. 2011. Bacterial community structures are unique and resilient in full-scale bioenergy systems. Proc. Natl. Acad. Sci. USA 108: 4158-4163.

15. Zhang T, Shao MF, Ye L. 2012. 454 Pyrosequencing reveals bacterial diversity of activated sludge from 14 sewage treatment plants. ISME J. 6: 1137-1147.

16. Nielsen PH. 2015. The activated sludge ecosystem contains a core community of abundant organisms. ISME J. 10: 11-20.

17. Riviere D, Desvignes V, Pelletier E, Chaussonnerie S, Guermazi S, Weissenbach J, et al. 2009. Towards the definition of a core of microorganisms involved in anaerobic digestion of sludge. ISME J. 3: 700-714.

18. Lee S-H, Kang H-J, Lee YH, Lee TJ, Han K, Choi Y, et al. 2012. Monitoring bacterial community structure and variability in time scale in full-scale anaerobic digesters. $J$. Environ. Monit. 14: 1893-1905.

19. de los Reyes FL, Weaver JE, Wang L. 2015. A methodological framework for linking bioreactor function to microbial communities and environmental conditions. Curr. Opin. Biotechnol. 33: 112-118.

20. APHA/AWWA/WEF. 2005. Standard Methods for the Examination of Water and Wastewater. American Public Health Association, the American Water Works Association, and the Water Environment Federation, Washington, DC.

21. Schloss PD, Westcott SL, Ryabin T, Hall JR, Hartmann M, Hollister EB, et al. 2009. Introducing mothur: open-source, platform-independent, community-supported software for describing and comparing microbial communities. Appl. Environ. Microbiol. 75: 7537-7541.

22. Hammer $\varnothing$, Harper DAT, Ryan PD. 2001. PAST: Paleontological Statistics Software Package for education and data analysis. Palaeontolia Electronica 4: 1-9.

23. Krzywinski M, Schein J, Birol I, Connors J, Gascoyne R, Horsman D, et al. 2009. Circos: an information aesthetic for comparative genomics. Genome Res. 19: 1639-1645.

24. Parks DH, Tyson GW, Hugenholtz P, Beiko RG. 2014. STAMP: statistical analysis of taxonomic and functional profiles. Bioinformatics 30: 3123-3124.

25. Stone L, Roberts A. 1990. The checkerboard score and species distributions. Oecologia 85: 74-79.

26. Barberan A, Bates ST, Casamayor EO, Fierer N. 2012. Using network analysis to explore co-occurrence patterns in soil microbial communities. ISME J. 6: 343-351.

27. Bastian M, Heymann S, Jacomy M. 2009. Gephi: an open source software for exploring and manipulating networks, pp.361-362. International AAAI Conference on Web and Social Media, North America, March 2009. Available at https://www.aaai.org/ocs/index.php/ICWSM/09/paper/view/ $154 / 1009$.

28. Chen Z, Wang H, Chen Z, Ren N, Wang A, Shi Y, et al. 2011. Performance and model of a full-scale up-flow anaerobic sludge blanket (UASB) to treat the pharmaceutical wastewater containing 6-APA and amoxicillin. J. Hazard. Mater. 185: 905-913.

29. Young JC, McCarty PL. 1969. The anaerobic filter for waste treatment. J. Water Pollut. Control Fed. R160-R173.

30. Wang C, Zuo J, Chen X, Xing W, Xing L, Li P, et al. 2014. Microbial community structures in an integrated two-phase anaerobic bioreactor fed by fruit vegetable wastes and wheat straw. J. Environ. Sci. 26: 2484-2492.

31. Liang B, Wang L-Y, Mbadinga SM, Liu J-F, Yang S-Z, Gu JD, et al. 2015. Anaerolineaceae and Methanosaeta turned to be the dominant microorganisms in alkanes-dependent methanogenic culture after long-term of incubation. $A M B$ Express 5: 1-13.

32. Hugenholtz P, Hooper SD, Kyrpides NC. 2009. Focus: Synergistetes. Environ. Microbiol. 11: 1327-1329.

33. Ariesyady HD, Ito T, Okabe S. 2007. Functional bacterial and archaeal community structures of major trophic groups in a full-scale anaerobic sludge digester. Water Res. 41: 1554-1568. 
34. Zhang Y, Wang X, Hu M, Li P. 2015. Effect of hydraulic retention time (HRT) on the biodegradation of trichloroethylene wastewater and anaerobic bacterial community in the UASB reactor. Appl. Microbiol. Biotechnol. 99: 1977-1987.

35. Carballa M, Regueiro L, Lema JM. 2015. Microbial management of anaerobic digestion: exploiting the microbiome-functionality nexus. Curr. Opin. Biotechnol. 33: 103-111.

36. Shen P, Zhang J, Zhang J, Jiang C, Tang X, Li J, et al. 2013. Changes in microbial community structure in two anaerobic systems to treat bagasse spraying wastewater with and without addition of molasses alcohol wastewater. Bioresour. Technol. 131: 333-340.

37. Lee C, Kim J, Chinalia FA, Shin SG, Hwang S. 2009. Unusual bacterial populations observed in a full-scale municipal sludge digester affected by intermittent seawater inputs. J. Ind. Microbiol. Biotechnol. 36: 769-773.

38. Shu D, He Y, Yue H, Wang Q. 2015. Microbial structures and community functions of anaerobic sludge in six fullscale wastewater treatment plants as revealed by 454 highthroughput pyrosequencing. Bioresour. Technol. 186: 163-172.

39. Chaffron S, Rehrauer H, Pernthaler J, von Mering C. 2010. A global network of coexisting microbes from environmental and whole-genome sequence data. Genome Res. 20: 947-959.

40. Lee C, Kim J, Hwang K, O'Flaherty V, Hwang S. 2009. Quantitative analysis of methanogenic community dynamics in three anaerobic batch digesters treating different wastewaters. Water Res. 43: 157-165. 